PAPER

\title{
Lower urinary tract function in patients with pituitary adenoma compressing hypothalamus
}

\author{
T Yamamoto, R Sakakibara, T Uchiyama, Z Liv, T Ito, T Yamanishi, T Hattori
}

J Neurol Neurosurg Psychiatry 2005;76:390-394. doi: 10.1136/jnnp.2004.044644

See end of article for authors' affiliations

.....................

Correspondence to: Dr Ryuji Sakakibara, Neurology Department, Chiba University, 1-8-1 Inohana Chuo-ku, Chiba 260-8670, Japan; sakakibara@faculty. chiba-u.jp

Received 2 May 2004

In revised form

15 June 2004

Accepted 25 June 2004
Background: The micturition reflex is under the tonic influence of suprapontine structures including the anteromedial frontal cortex, basal ganglia, and hypothalamus. However, there have been few reports about the role of the hypothalamus on the lower urinary tract (LUT) function in humans.

Objective: To investigate LUT function in patients with pituitary adenomas.

Methods: Urodynamic studies were carried out in three patients with LUT symptoms who had pituitary adenomas extending upwards to the hypothalamus.

Results: All three male patients (age 28 to 62 years) developed LUT symptoms (urinary urgency and frequency (3); urinary incontinence (3); voiding difficulty and retention (2)) along with weight loss, psychiatric symptoms, unsteady gait, and/or visual disturbances. One had the syndrome of inappropriate secretion of antidiuretic hormone, but none had diabetes insipidus. Two had resection of the tumour and subsequent radiation therapy, but LUT dysfunction persisted. The third patient had partial resection of the tumour to ameliorate hydrocephalus. Urodynamic studies showed detrusor overactivity during the storage phase in all patients; during the voiding phase there was underactive detrusor in two and non-relaxing sphincter in one.

Conclusions: Hypothalamic lesions can cause severe LUT dysfunction in both the storage and voiding phases of micturition. This may reflect the crucial role of the hypothalamus in regulating micturition in humans.
$\mathrm{L}$ ower urinary tract (LUT) function is dependent on the spino-bulbo-spinal reflex arc in both experimental animals ${ }^{1}$ and humans. ${ }^{23}$ The micturition reflex is under the tonic (mainly inhibitory) influence of several suprapontine structures, including the anteromedial frontal cortex (frontal micturition centre), ${ }^{45}$ the basal ganglia, ${ }^{6}$ and the hypothalamus. ${ }^{7-9}$ However, there have been few reports on clinical LUT dysfunction resulting from hypothalamic lesions. ${ }^{10-12}$ Ten years ago we had two such patients who presented with LUT dysfunction. Each had a pituitary adenoma extending upwards to the hypothalamus, which was detected by brain computed tomography (CT) with positive contrast enhancement. ${ }^{12}$ Recently, we encountered a third patient with the same brain lesion, detected by a magnetic resonance imaging (MRI). In this report, we describe the LUT function of these three patients, with a brief review of the literature.

\section{CASE REPORT}

Case 1

A 28 year old previously healthy man began to have progressive anorexia and weight loss $(86 \mathrm{~kg}$ to $61 \mathrm{~kg}$ within four months). A month later, his family noticed that he had night time urinary frequency, urinary urgency, and dysarthria. In the next month, he was admitted to a local gastroenterology hospital. Fibreoptic examination of both upper and lower gastrointestinal tracts was carried out, but the results were normal. On admission to our department the next month, his LUT symptoms included night time urinary frequency (two to three times), urinary urgency, and reflex urinary incontinence (once or twice a day), but without daytime urinary frequency, nocturnal enuresis, or difficulty in voiding. He had no episodes of postural hypotension or constipation.

On examination, he was markedly emaciated $(172 \mathrm{~cm}$; $54.5 \mathrm{~kg}$ ). His conjunctivae were pale, and his skin was dry. His pubic hair was thin. Mild gynaecomastia was observed, and the development of the penis and testes was poor. His state of consciousness was normal. However, he was depressive and a suicidal tendency was suspected. He had bitemporal hemianopia. Other cranial nerves were normal except for mild dysarthria. He showed bradykinesia and muscle rigidity, along with mild bilateral weakness of the flexor muscles in the lower extremities. The deep tendon reflexes were brisk, but Hoffman and Babinski signs were negative. Sensory examination was normal, including the genital area.

Routine laboratory investigations showed mild sideropenic anaemia. Cerebrospinal fluid examination revealed a mildly increased cell count of $7 / \mathrm{mm}^{3}$ (all mononuclear cells; no malignant cells were seen), a total protein of $85 \mathrm{mg} / \mathrm{dl}$, but normal values of glucose $(2.94 \mathrm{mmol} / \mathrm{l})$ and chloride ( $126 \mathrm{mmol} / \mathrm{l})$. Endocrine examination showed low values in serum testosterone ( $1.08 \mathrm{ng} / \mathrm{ml})$, oestrogen El (20.3 IU/l), E2 $(<10 \mathrm{IU} / \mathrm{l})$, and E3 $(<5 \mathrm{IU} / \mathrm{l})$. Baseline levels of thyroid stimulating hormone (TSH), adrenocorticotropic hormone $(\mathrm{ACTH})$, growth hormone $(\mathrm{GH})$, and prolactin (PRL) were normal. However, tolerance tests showed low secretion of hormones in responses to insulin, thyroid hormone releasing hormone (TRH), bromocriptine, and luteinising hormone releasing hormone (LHRH). Serum antidiuretic hormone $(\mathrm{ADH})$ was normal and there were no signs of diabetes insipidus or nocturnal polyuria. Analysis of the chromosomal pattern showed 47XXY. Brain CT revealed a pituitary tumour $(8 \times 8 \times 20 \mathrm{~mm})$ extending upward to the hypothalamus with positive contrast enhancement, which seemed to compress the hypothalamus, optic chiasm, stria terminalis, septal area, and supraoptic area (fig 1A). A diagnosis of Klinefelter's syndrome was made on the basis of the physical signs,

Abbreviations: LUT, lower urinary tract; PAG, periaqueductal grey matter; PMC, pontine micturition centre; SIADH, syndrome of inappropriate secretion of antidiuretic hormone 

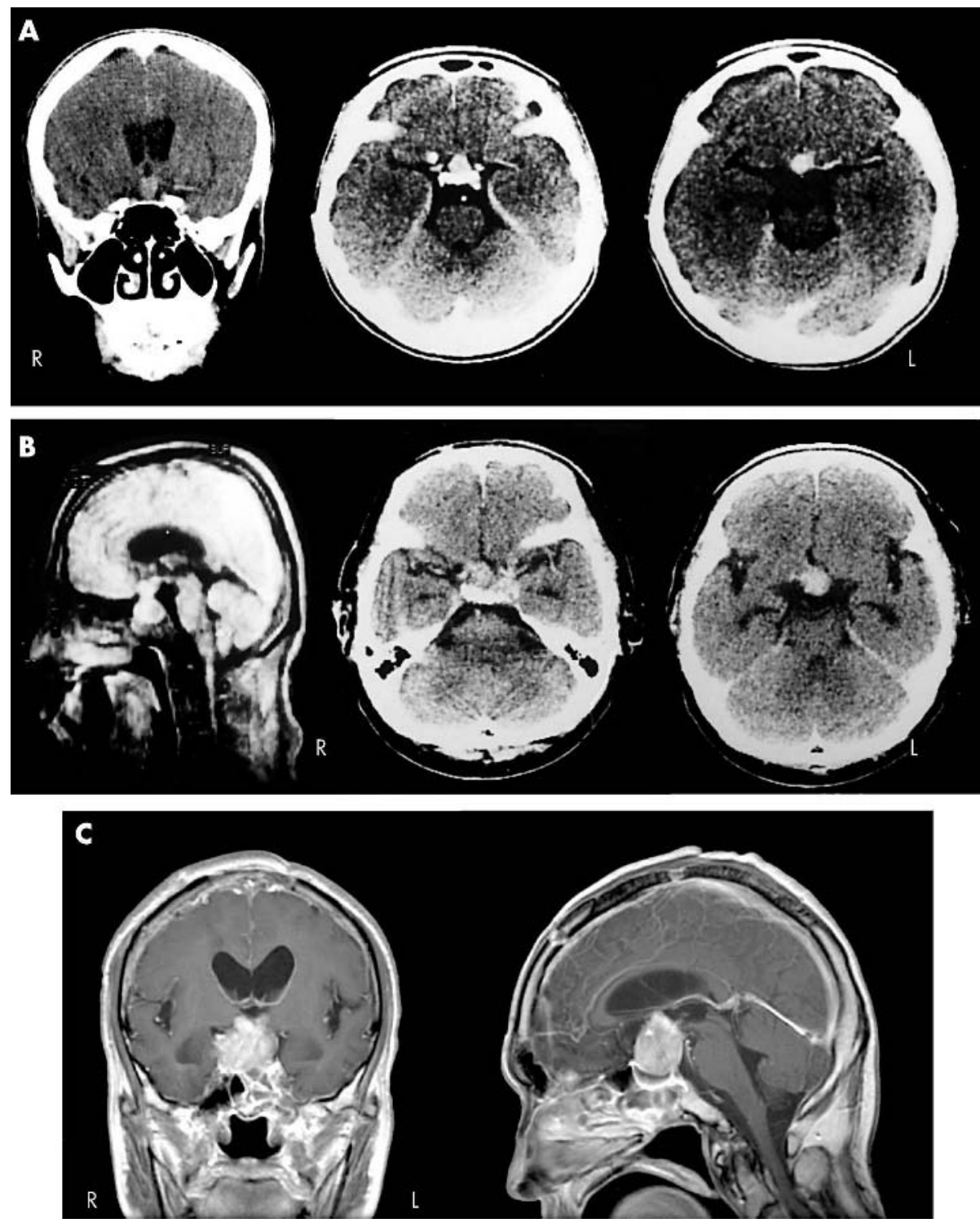

Figure 1 Brain imaging in the patients described. (A) Brain computed tomography (CT) of case 1 (coronal and axial planes with contrast enhancement), showing a pituitary tumour $(8 \times 8 \times 20 \mathrm{~mm})$ extending upward to the hypothalamus, seeming to compress the hypothalamus, optic chiasm, stria terminalis, septal area, and supraoptic area. (B) Magnetic resonance imaging (MRI) (sagittal plane, proton weighted image) and CT (axial plane with contrast enhancement) of case 2, showing a similar sized pituitary tumour ( $10 \times 10 \times 25 \mathrm{~mm}$ ). (C) MRI in case 3 (coronal and sagittal planes, gadolinium-DTPA images), showing a pituitary tumour $(35 \times 25 \times 20 \mathrm{~mm})$ extending upward to the third ventricle and compressing the hypothalamus bilaterally.

endocrine examination, and chromosomal pattern. In addition, the rapid progressive weight loss, psychiatric symptoms, visual field defect, and the LUT dysfunction were thought to be caused by the pituitary adenoma compressing the hypothalamus.

Subtotal resection of the tumour and subsequent radiation therapy (total 61 Gy) were undertaken. Pathology revealed a pituitary adenoma. As a result, though visual field disturbance continued to progress mildly, the weight loss halted and the psychiatric symptoms improved. However, nocturnal urinary frequency and urge incontinence persisted, so urodynamic studies were carried out. Rectal examination and urethrocystography showed no evident prostatic hyperplasia or urethral stricture. He had no urinary tract infection at the time of urodynamic studies, nor was he taking a drug that would influence LUT function. The methods and definitions used for the urodynamic studies conformed to the standards proposed by the International Continence Society. ${ }^{13}$ The normal range of urodynamic variables is as follows:

- residual urine volume under $30 \mathrm{ml}$;

- first sensation more than $100 \mathrm{ml}$ but less than $300 \mathrm{ml}$;

- bladder capacity more than $200 \mathrm{ml}$ but less than $600 \mathrm{ml}$.

After voluntary voiding, residual urine measurement in case 1 showed a minimum post-void residual volume of $40 \mathrm{ml}$. Electromyography (EMG)-cystometry was undertaken with an $8 \mathrm{~F}$ double lumen urethral catheter, a rectal catheter, and a concentric needle electrode that was inserted into the anal sphincter. He had normal first sensation of $100 \mathrm{ml}$, but a small maximum bladder capacity of $140 \mathrm{ml}$. Detrusor overactivity was noted at the end of bladder filling. When asked to void, following the detrusor overactivity his detrusor 
pressure increased moderately. There was no detrusorsphincter dyssynergia. These results indicated the presence of neurogenic LUT dysfunction.

\section{Case 2}

A 62 year old man began to have mild fever $\left(38.0^{\circ} \mathrm{C}\right)$, general weakness, and symptoms of upper respiratory infection. A week later, administration of an anti-inflammatory drug improved his pyrexia. However, general weakness progressed and he developed gait disturbance. He also experienced voiding difficulty, but not urinary frequency, urgency, or incontinence. He had no episodes of postural hypotension or constipation. A week later, he was admitted to our hospital.

Physical examination on admission showed no abnormal findings. Neurological examination showed some degree of disorientation in time and space. He had enlargement of the blind spot and temporal narrowing of the visual fields bilaterally. The other cranial nerves were normal. There was mild weakness of the left arm and leg. The deep tendon reflexes were exaggerated, but Hoffman and Babinski signs were not observed. Sensation, including that in the genital area, was normal.

Two days after admission, he suddenly developed urinary retention without any precipitating cause, and an indwelling balloon catheter was inserted. Routine laboratory tests were normal except for marked hyponatraemia ( $\mathrm{Na} 114 \mathrm{mmol} / \mathrm{l}$ ) and low serum chloride $(82 \mathrm{mmol} / \mathrm{l})$. Endocrine examination showed that basic secretion of most of the anterior pituitary hormones was decreased, but secretion of posterior pituitary hormone $(\mathrm{ADH})$ was increased. A diagnosis of the syndrome of inappropriate secretion of $\mathrm{ADH}$ (SIADH) was therefore made. Brain CT and MRI showed a pituitary tumour $(10 \times 10 \times 25 \mathrm{~mm})$ extending upward to the hypothalamus with positive contrast enhancement (fig 1B). The tumour extended from the pituitary gland to the optic chiasm, hypothalamus, stria terminalis, septal area, and preoptic area. Cervical $x$ ray showed mild spondylotic change in the third and fourth vertebrae. On rectal examination and urethrocystography there was no evident prostatic hyperplasia or urethral stricture. He had no urinary tract infection at the time of urodynamic studies, nor was he taking any drug that would influence LUT function.

On EMG-cystometry, the first sensation was $230 \mathrm{ml}$ and the maximum bladder capacity was $510 \mathrm{ml}$ (both in the normal range). Minor detrusor overactivity was observed during bladder filling. When asked to void, following slight detrusor overactivity, he was unable to contract the detrusor at all (underactive detrusor). He could not relax the sphincter on voiding. These results indicated neurogenic LUT dysfunction.

After admission to our hospital, his visual disturbance gradually progressed, and he developed bitemporal hemianopia. Though he could void by himself, micturition difficulty persisted and night time urinary frequency (two to three times) and urinary urgency (two to three times a week) became apparent. We considered that hyposecretion of anterior pituitary hormones, SIADH, visual field disturbance, and the LUT dysfunction were likely to be caused by the pituitary adenoma compressing the hypothalamus. We prescribed $20 \mathrm{mg} /$ day of prednisolone to counter the anterior pituitary hyposecretion, and his water intake was limited to $800 \mathrm{ml} /$ day to ameliorate the SIADH. Partial resection of tumour and subsequent radiation therapy (total $40 \mathrm{~Gy}$ ) were carried out. Pathology of the resected specimen showed a pituitary adenoma. Following surgery, his visual disturbance improved somewhat on the left and he became able to urinate. However, right sided visual disturbance persisted, and he still had night time frequency (two to three times), urinary urgency (two to three times a week), and mild voiding difficulty.

\section{Case 3}

A 60 year old man gradually developed an unsteady gait, which was followed by disorientation, urinary urgency/ frequency, and voiding difficulty which were present for four months before his admission to hospital. On admission to our hospital, his LUT symptoms included daytime frequency (10 times), night time frequency (four times), urinary urgency, and urgency/functional incontinence, but without nocturnal enuresis, delay in initiating urination, prolongation of urination, straining, or the sensation of postvoid residual urine. He had no postural hypotension or constipation.

On examination, he was uncooperative and had disorientation and dementia. On Hasegawa's dementia rating scale (equivalent to the mini-mental state examination), he scored $15 / 30$. He was also uncooperative to complete visual field examination. He had no apparent motor weakness. Deep tendon reflexes were normal, with negative Babinski sign. He had mild postural tremor and rigidity in both hands. There was a slight lack of coordination and a mildly broad based gait. Sensation was normal. Routine laboratory data were normal. Gonadotropins (LH, FSH, prolactin), GH, and TSH were normal. He did not have diabetes insipidus.

Brain MRI showed a pituitary mass $(35 \times 25 \times 20 \mathrm{~mm})$ which extended upward to the hypothalamus and appeared with low signal intensity on Tl weighted images and with high signal intensity on T2 weighted images. GadoliniumDTPA images showed positive contrast enhancement. Brain MRI also showed mildly enlarged lateral ventricles (secondary hydrocephalus), most probably caused by obstruction of the foramina of Monro by the mass lesion.

He underwent partial resection of the tumour, which completely reversed the hydrocephalus and gradually ameliorated his disorientation and unsteady gait. Pathology of the resected specimen revealed a pituitary adenoma. However, postoperatively he became unable to void and had to begin clean intermittent catheterisation (CIC) three times a day. His voided volume was 30 to $50 \mathrm{ml}$ and the volume of post-void residual urine was 300 to $600 \mathrm{ml}$, showing urinary retention. The follow up brain MRI two weeks after surgery revealed tumour in the third ventricle still compressing the hypothalamus bilaterally (fig lC). There was no evidence of prostatic hypertrophy on rectal examination or ultrasound echography. He had no urinary tract infection at the time of the urodynamic studies, nor was he taking a drug that would influence LUT function.

Urodynamic studies showed a voluntary voided volume of $80 \mathrm{ml}$ with a low maximum flow rate (Qmax) of $4 \mathrm{ml} / \mathrm{s}$ and a low average flow rate (Qave) of $2 \mathrm{ml} / \mathrm{s}$. He had a post-void residual volume of $220 \mathrm{ml}$. On EMG-cystometry, the first sensation was at $220 \mathrm{ml}$ and the maximum bladder capacity was $235 \mathrm{ml}$, both in the normal range. However, detrusor overactivity was noted at the end of bladder filling. When asked to void, following the detrusor overactivity, his detrusor pressure (Pdet) increased only slightly, with poor urinary flow. There was no detrusor-sphincter dyssynergia. We also carried out a pressure-flow analysis. The peak Pdet at Qmax (PdetQmax) indicated equivocal obstruction by the Abrams-Griffiths' nomogram, ${ }^{14}$ and grade 2 (equivocal obstruction) with weak detrusor contraction by Schäfer's nomogram..$^{15}$ These results indicated the presence of neurogenic LUT dysfunction.

To ameliorate the voiding difficulty, the patient was started on $2 \mathrm{mg} /$ day of oral urapidil hydrochloride, a selective $\alpha_{1}$ adrenergic antagonist. Two weeks after starting this treatment, the voiding difficulty had improved and the voiding 
parameters were now as follows: voided volume, $110 \mathrm{ml}$; Qmax, $13 \mathrm{ml} / \mathrm{s}$; Qave, $7 \mathrm{ml} / \mathrm{s}$; Schäfer grade l (normal/ equivocal obstruction); weak detrusor contraction; residual urine volume, $10 \mathrm{ml}$.

\section{DISCUSSION}

Until recently, few reports have been available on the role of the hypothalamus in LUT function in humans. ${ }^{10-12}$ This is probably because a critical illness such as disturbance of consciousness in the patients with hypothalamic lesions could easily mask any LUT dysfunction that might be present. In 1950 , Brouwer ${ }^{10}$ reported a patient initially presenting with urinary incontinence. Necropsy proved that he had a glioma located in the hypothalamus and the third ventricle. In 1965, Andrew and Nathan ${ }^{11}$ described a patient presenting with loss of visual acuity, urinary frequency, urge urinary incontinence, and nocturnal enuresis. Surgical exposure of the brain revealed a cystic lesion compressing the optic chiasm, anterior hypothalamus, and septal area. In the following year, Andrew and Nathan ${ }^{16}$ reported five patients with LUT symptoms appearing after a ruptured cerebral aneurysm. All patients had motor paresis or exaggerated deep tendon reflexes. Surgical procedures carried out in these patients included ligation of the anterior cerebral artery and resection of the frontal rectal gyrus. Although these investigators speculated that the site of lesion responsible for the LUT dysfunction was the anterior hypothalamus, the lesions seemed to be much more widespread than the anterior hypothalamus alone.

Our three patients had various combinations of LUT symptoms (case 1, nocturnal frequency, urinary urgency, reflex incontinence; case 2, nocturnal frequency, urgency incontinence, voiding difficulty, and urinary retention; case 3, diurnal and nocturnal frequency, urgency incontinence, and urinary retention), together with visual disturbances, anorexia, psychiatric symptoms, and SIADH. Case 1 had Klinefelter syndrome, and case 2 had mild cervical spondylosis at the C3 and 4 vertebrae. However, the LUT symptoms appeared along with symptoms suggestive of hypothalamic lesions. In case 3, LUT symptoms were accompanied by disorientation and gait difficulty caused by secondary hydrocephalus. After resolution of the hydrocephalus following partial resection of the tumour, the patient's disorientation and gait difficulty improved. However, the LUT symptoms persisted and he developed complete urinary retention. None of the three patients had diabetes insipidus (polyuria) from posterior pituitary insufficiency. Rectal examination, cystourethrography, and ultrasound echography showed no evidence of prostatic hyperplasia. They had no urinary tract infections and were taking no drugs that would influence LUT function. Brain MRI or CT in each case revealed a mass lesion in the pituitary gland and the hypothalamus extending to the optic chiasm, stria terminalis, septal region, and the preoptic area. Pathology revealed a pituitary adenoma in all cases. Thus the LUT symptoms in our patients can be attributed to the pituitary adenoma compressing the hypothalamus.

Information arising from the LUT reaches the midbrain periaqueductal grey matter (PAG), then descends back to the pontine micturition centre (PMC), which activates the descending pathway to the sacral parasympathetic preganglionic neurones innervating the bladder (pelvic nerves). ${ }^{1}$ The spino-bulbo-spinal micturition reflex is under tonic (mainly inhibitory) influence from several suprapontine structures including the hypothalamus. ${ }^{7-9}$ It is well known that cerebral diseases can lead to a loss of the brain's inhibitory influence on the micturition reflex. ${ }^{17}$ Filling phase cystometry in the present study showed detrusor overactivity - which is a phasic increase in the detrusor pressure-in all three patients studied. Detrusor overactivity is the major cause of storage disorder. ${ }^{17}$ Previously, Andrew and Nathan ${ }^{4}$ also described detrusor overactivity in a patient with a third ventricular cyst. In an experimental setting, chemical stimulation of the medial hypothalamus in freely moving rats elicits defencelike reactions such as locomotion, rearing, and micturition, ${ }^{18}$ which are akin to those elicited by PAG stimulation (the emotional motor system)..$^{19}{ }^{20}$ This is presumably because the hypothalamus has fibre connections with the limbic brain (the anterior frontal cortex, cingulate cortex, and amygdala). ${ }^{21}$ Under anaesthesia, electrical stimulation of the hypothalamus in cats and dogs elicits either facilitation (particularly in the anterolateral part and the preoptic area) or inhibition (in the medial part and Forel's $\mathrm{Hl}$ area) of the micturition reflex. ${ }^{7-9}$ In our three patients, however, we could not analyse the detailed location of lesions within the hypothalamus.

During urinary storage, the hypothalamic neurones are known to be activated in both experimental animals ${ }^{22}$ and humans. ${ }^{23}$ The hypothalamus and the preoptic area also have dense fibre connections with the PAG and the PMC, ${ }^{24-26}$ both of which are crucial for the spino-bulbo-spinal micturition reflex arc. The hypothalamus also projects to Onuf's (sacral) nucleus, which innervates the urethral sphincter muscles (pudendal nerves). ${ }^{9}$ During the voiding phase, two of our patients (cases 2 and 3) showed an underactive detrusor, though both patients had detrusor overactivity during bladder filling. This condition is known to occur in various types of neurogenic bladder dysfunction. ${ }^{27}$ In addition, one patient (case 2) had an unrelaxing sphincter on voiding, though it normally relaxes completely, ${ }^{17}$ which suggests incoordinated neural control of the bladder and urethra. ${ }^{17}$ Hence, a lesion in the hypothalamus may cause various types of LUT dysfunction, reflecting the crucial role of the hypothalamus in regulating micturition in humans.

\section{Conclusions}

Our results show that pituitary adenomas compressing the hypothalamus can cause severe LUT dysfunction in both the storage and the voiding phases, reflecting the crucial role of the hypothalamus in regulating micturition in humans.

\section{Authors' affiliations}

T Yamamoto, R Sakakibara, T Uchiyama, Z Liu, T Ito, T Hattori,

Department of Neurology, Chiba University, Chiba, Japan

T Yamanishi, Department of Urology, Dokkyo Medical College, Tochigi, Japan

Competing interests: none declared

\section{REFERENCES}

1 de Groat WC, Booth AM, Yoshimura N. Neurophysiology of micturition and its modification in animal models of human disease. In: Maggi CA, ed. Nervous control of the urogenital system, the autonomic nervous system, vol. 3. London: Harwood Academic Publisher, 1993:227-90.

2 Sakakibara R, Hattori T, Yasuda K, et al. Micturitional disturbance and pontine tegmental lesion; urodynamic and MRI analyses of the vascular cases. J Neurol Sci 1996;141:105-10.

3 Aswal BS, Berkley KJ, Hussain I, et al. Brain responses to changes in bladder volume and urge to void in healthy men. Brain 2001;124:369-77.

4 Gjone R. Excitatory and inhibitory bladder responses to stimulation of "limbic", diencephalic and mesencephalic structures in the cat. Acta Physiol Scand 1966;66:91-102.

5 Andrew J, Nathan PW. Lesions of the anterior frontal lobes and disturbances of micturition and defaecation. Brain 1964;87:233-62.

6 Sakakibara R, Nakazawa K, Uchiyama T, et al. Effect of subthalamic nucleus stimulation on the Micturition reflex in cats. Neuroscience 2003;120:871-5.

7 Enoch DM, Kerr FWL. Hypothalamic vasopressor and vesicopressor pathways. I. Functional studies. Arch Neurol 1967;16:290-306.

8 Enoch DM, Kerr FWL. Hypothalamic vasopressor and vesicopressor pathways. Il. Anatomic study of their course and connections. Arch Neurol 1967; 16:307-20.

9 Yamane M. Effects of hypothalamic stimulation on oufflow of the pelvic vesical and rectal branches in dogs. Auton Nerv Syst 1989;26:563-73. 
10 Brouwer B. Positive and negative aspects of hypothalamic disorders. J Neurol Neurosurg Psychiatry 1950;13:16-23.

11 Andrew J, Nathan PW. The cerebral control of micturition. Proc R Soc Med 1965;58:553-5.

12 Sakakibara $\mathbf{R}$, Hattori T, Tojo $M$, et al. Micturitional disturbance in patients with pituitary-hypothalamic lesions. Auton Nerv Syst 1994;31:717-22. [In Japanese with English abstract].

13 Abrams P, Cardozo L, Fall M, et al. The standardization of terminology of lower urinary tract function: report from the standardization sub-committee of the international continence society. Neurourol Urodynam 2002;21:167-78.

14 Griffiths D, Höfner K, van Mastrigt R, et al. Standardization of terminology of lower urinary tract function; pressure-flow studies of voiding, urethral resistance, and urethral obstruction. Neurourol Urodynam 1997;16:1-18.

15 Schäfer W. Principles and clinical application of advanced urodynamic analysis of voiding dysfunction. Urol Clin North Am 1990;17:553-66.

16 Andrew J, Nathan PW. Disturbances of micturition and defaecation due to aneurysms of anterior communicating or anterior cerebral arteries. J Neurosurg 1966;24:1-10.

17 Blaivas JG. The neurophysiology of micturition; a clinical study of 550 patients. J Urol 1982;127:958-63.

18 Silveira MC, Graeff FG. Defense reaction elicited by microinjection of kainic acid in the medial hypothalamus of the rat. Brazilian J Med Biol Res 1988;21:569-71.
19 Vargas LC Marques TD, Schenberg LC. Micturition and defensive behaviors are controlled by distinct neural networks within the dorsal periaqueductal gray and deep gray layer of the superior colliculus of the rat. Neurosci Lett 2000;280:45-8.

20 Blok BFM. Holstege $G$. The neural control of micturition and its relation to the emotional motor system. Prog Brain Res 1996;107:113-26.

21 Brodal A. In: Neurological anatomy in relation to clinical medicine, 3rd ed. Oxford: Oxford University Press, 1981.

22 Stuart DG, Porter RW, Adey WR, et al. Hypothalamic unit activity. I. Visceral and somatic influences. Electrophysiol Clin Neurophysiol 1964:16:237-47.

23 Blok B, Willemsen T, Holstege G. A PET study of brain control of micturition in humans. Brain 1997; 129:111-21.

24 Holstege G. Some anatomical observations on the projections from the hypothalamus to brainstem and spinal cord; an HRP and autoradiographic tracing study in the cat. J Comp Neurol 1987; 260:98-126.

25 Rizvi TA, Murphy AZ, Ennis $M$, et al. Fos expression in rat pontine tegmental neurons following activation of the medial preoptic area. Brain Res 1998:789:256-62.

26 Ding $Y Q$, Wang $D, X u J Q$, et al. Direct projections from the medial preoptic area to spinally-(projecting) neurons in Barrington's nucleus: an electron microscope study in the rat. Neurosci Lett 1999;271:175-8.

27 Resnick NM, Yalla SV. Detrusor hyperactivity with impaired contractile function. JAMA 1987;257:3076-81. 